\title{
EXISTENCE THEOREMS FOR THE IMPLICIT COMPLEMENTARITY PROBLEM
}

\author{
G. ISAC \\ Département de Mathématiques \\ Collége Militaire Royal \\ St-Jean, Québec, Canada \\ JOJ 1RO \\ and \\ D. Goeleven
}

Département de Mathématiques

Faculté des Sciences

Université de Namur

Rempart de la Vierge 8

B-5000 Namur, Belgique

(Received August 20, 1991 and in revised form April 16, 1992) ABSTRACT. Some existence theorems for the general implicit complementarity problem in an
infinite dimensional space are considered.

KEY WORDS AND PHRASES. Complementarity Problem, Existence Theorems. 1991 AMS SUBJECT CLASSIFICATION CODES. $49 J 40$.

\section{INTRODUCTION.}

The study of Complementarity Problems is an interesting and important domain of applied mathematics [1], [5], [8], [10] etc. In this domain, a special chapter is the Implicit Complementarity Problem. It seems that the first Implicit Complementarity Problem was defined in 1973 by Bensoussan and Lions [2], as the mathematical model of some stochastic optimal control problems [2], [3], [4]. Now, it is well known that, the Implicit Complementarity Problem can be used to study the optimal stopping of Markov chains [6].

The first existence results for the Implicit Complementarity are the results obtained by Dolcetta and Mosco [7], [18], [19].

As numerical methods for solving the Implicit Complementarity Problem we remark the iterative methods proposed by Pang [20], [21] and Mosco [22].

In this paper, we study some existence theorems for the general Implicit Complementarity Problem in an infinite dimensional space. This paper can be considered as a complement of our paper [13].

2. DEFINITION OF PROBLEM AND PRELIMINARIES.

Let $\left\langle E, E^{*}\right\rangle$ be a dual system of Banach spaces. Denote by $K$ a pointed convex cone in $E$, that is, a subset of $E$ satisfying the following properties:

$\left.\left.1^{\circ}\right) K+K \subseteq K 2^{\circ}\right) \lambda K \subseteq K$, for all $\lambda \in R$ and $\left.3^{\circ}\right) K \cap(-K)=\{0\}$.

The closed convex cone $K^{*}=\left\{y \in C^{*} \mid\langle x, y\rangle \geq 0\right.$; for all $\left.x \in K\right\}$ is called the dual of $K$. 
Given a subset $D \subset E$ and the mappings $S: D \rightarrow K$ and $T: D \rightarrow E^{*}$, the Implict Complementarity Problem associated to $T, S$ and $\mathrm{K}$ is

$$
\operatorname{ICP}(T, S, \mathrm{~K}):\left\{\begin{array}{l}
\text { find } x_{o} \in D \text { such that } \\
T\left(x_{o}\right) \in K^{*} \text { and } \\
<S\left(x_{o}\right), T\left(x_{o}\right)>=0 .
\end{array}\right.
$$

We find applications and examples of this problem in [2], [3], [4], [6], [7], [18], [19], [20], [21]. When $D=K$ and $S(x)=x$, for all $x \in K$, the problem $\operatorname{ICP}(T, S, K)$ is exactly the nonlinear complementarity problem, which has interesting applications in: Optimization, Game Theory, Economics, Mechanics, etc. [1], [5], [8-15].

If the problem $\operatorname{ICP}(T, S, \mathrm{~K})$ is defined, we consider the following special variational inequality:

$$
S V I(T, S, K):\left[\begin{array}{l}
\text { find } x_{o} \in D \text { such that } \\
<x-S\left(x_{o}\right), T\left(x_{o}\right)>\geq 0 ; \forall x \in K .
\end{array}\right.
$$

PROPOSITION 1. The problem $S V I(T, S, \mathrm{~K})$ is equivalent to the problem $\operatorname{ICP}(T, S, \mathrm{~K})$.

PROOF. Indeed, if $x_{o}$ is a solution of the problem $S V I(T, S, K)$ then $S\left(x_{o}\right) \in K$ and we have

$$
(1):\left\langle x-S\left(x_{o}\right), T\left(x_{o}\right)>\geq 0 ; \forall x \in K\right.
$$

Let $u \in K$ be an arbitrary element. If we put $x=u+S\left(x_{o}\right)$ in (1) we obtain $\left\langle u, T\left(x_{o}\right)\right\rangle \geq 0$, for every $u \in K$, that is, we have $T\left(x_{o}\right) \in K^{*}$.

If we put $x=0$ in (1) we have $\left\langle S\left(x_{o}\right), T\left(x_{o}\right)\right\rangle \leq 0$ and since $\left\langle S\left(x_{o}\right), T\left(x_{o}\right)\right\rangle \geq 0$ we deduce $\left\langle S\left(x_{o}\right), T\left(x_{o}\right)>=0\right.$.

Conversely, let $x_{o}$ be a solution of the problem $\operatorname{ICP}(T, S, K)$. We have, $S\left(x_{o}\right) \in \mathrm{K}, T\left(x_{o}\right) \in K^{*}$ and $\left\langle S\left(x_{o}\right), T\left(x_{o}\right)\right\rangle=0$ which imply $\left\langle x-S\left(x_{o}\right), T\left(x_{o}\right)\right\rangle \geq 0$, for every $x \in K$.

Given a nonempty subset $D \subset E$ and the mappings $T: D \rightarrow E^{*}$ and $S: D \rightarrow K$ we consider the following problem

$$
S V I(T, S, D):\left[\begin{array}{l}
\text { find } x_{o} \in D \text { such that } \\
<x-S\left(x_{o}\right), T\left(x_{o}\right)>\geq 0 ; \forall x \in D,
\end{array}\right.
$$

To solve the problem $S V I(T, S, D)$ we use the following classical result.

THEOREM 1. A mapping $T_{o}: D \rightarrow 2 D$, where $D \subset X$, have a fixed point if the following conditions are satisfied:

$\left.1^{\circ}\right): \quad X$ is a locally convex space and the set $D$ is nonempty, compact, and convex,

$2^{\circ}$ ): the set $T_{o}(X)$ is nonempty and convex for all $x \in D$ and the preimages $T_{0}^{-1}(y)$ $=\left\{x \in D \mid y \in T_{o}(x)\right\}$ are relatively open with respect to $D$, for all $y \in D$.

PROOF. The proof is in [25][Proposition 9.9, p. 453].

THEOREM 2. Let $D \subset E$ be a nonempty compact convex set, $T: D \rightarrow E^{*}$ and $S: D \rightarrow K$ two continuous mappings.

If for every $x \in D$ we have $\langle S(x), T(x)\rangle \leq\langle x, T(x)\rangle$, then the problem $S V I(T, S, D)$ has a solution.

PROOF. If the problem $S V I(T, S, D)$ does not have a solution then,

$$
\text { (2): }(\forall x \in D)(\exists u \in D)(<u-S(x), T(x)><0)
$$


Let $T_{o}: D \rightarrow D$ be the point-to-set mapping defined by, $T_{o}(x)=\{u \in D \mid<u-S(x), T(x)><0\}$, for every $x \in D$.

We remark that $T_{o}(x)$ is nonempty and convex for every $x \in D$.

Since $T$ and $S$ are continuous, the mapping $v \rightarrow\langle x-S(v), T(v)\rangle$ is continuous too and we have that $T_{o}^{-1}(y)=\left\{x \in D \mid y \in T_{o}(x)\right\}=\{x \in D \mid<y-S(x), T(x)><0\}$ is relatively open with respect to $D$.

Hence, by Theorem 1 there is an element $x_{*} \in D$ such that $x_{*} \in T_{o}\left(x_{*}\right)$, that is, $<x_{*}-S\left(x_{*}\right)$, $T\left(x_{*}\right)><0$, which is impossible since for every $x \in D$ we have (by assumption) $<S(x)$, $T(x)\rangle \leq\langle x, T(x)>$.

Let $K$ be a pointed convex cone in $E$. We say that a subset $B$ of $K$ is a base, if $B$ is convex and for every $x \in K \backslash\{0\}$ there is a unique $b_{x} \in B$ and a unique number $\lambda_{x} \in R+\backslash\{0\}$ such that $x=\lambda_{x} b_{x}$.

A closed pointed convex cone $K \subset E$ is locally compact if and only if, it has a compact base [Klee's Theorem].

If $r \in \mathbf{R}_{+} \backslash\{0\}$ we denote $K_{r} \leq=\{x \in K \mid\|x\| \leq r\}$ and $K_{r}{ }^{<}=\{x \in K \mid\|x\|<r\}$.

We say that a convex cone $K \subset E$ is a Galerkin cone [10] if there exists a countable family of convex subcones $\left\{K_{n}\right\}_{n \in N}$ of $K$ such that:

i) $K_{n}$ is locally compact for every $n \in N$,

ii) if $n \leq m$ then $K_{n} \subseteq K_{m}$,

iii) $K=\overline{=} \underset{n \in N}{\bigcup_{n} K_{n}}$

A Galerkin cone will be denoted by $K\left(K_{n}\right)_{n \in N}$.

We recall that if $D \subset E$ is a closed convex set, we say that a continuous operator (not necessary linear) $P: E \rightarrow E$ is a projection onto $D$ if $P(E)=D$ and $P(x)=x$ for every $x \in D$.

By the same proof as in our paper [12] we can prove that if $K\left(K_{n}\right)_{n \in N}$ is a Galerkin cone in a Banach space, then for every $n \in N$ there exists a projection $P_{n}$ onto $K_{n}$ such that for every $x \in K$ we have $\lim _{n \rightarrow \infty} P_{n}(x)=x$.

Given two Banach spaces $(E,\|\|)$ and $(F,\|\|)$ we say that an operator (not necessary linear) $T: E \rightarrow F$ is strongly continuous if for every sequence $\left\{x_{n}\right\}_{n \in N} \subset E$, weakly convergent to $x_{*}$ we have that $\left\{T\left(x_{n}\right)\right\}_{n \in N}$ is norm convergent to $T\left(x_{*}\right)$.

This class of operators is very important and was intensively studied by Vainberg [24] and Lipkin [17].

3. PRINCIPAL RESULTS.

The principal aim of this paper is to give some existence theorems for the problem $\operatorname{ICP}(T, S, \mathrm{~K})$.

In this sense, we suppose given a dual system $\left\langle E, E^{*}\right\rangle$ of Banach spaces. We consider on $E^{*}$ the strong topology.

THEOREM 3. Let $K \subset E$ be a pointed locally compact cone and $S: K \rightarrow E, T: K \rightarrow E^{*}$ continuous mappings. If the following assumptions are satisfied:

$\left.1^{\circ}\right)$ there is a number $r>0$ such that $S\left(K_{r} \leq\right) \subseteq K$,

$\left.2^{\circ}\right)$ there is an element $u_{o} \in K$ such that $S\left(u_{o}\right) \in K,\left\|S\left(u_{o}\right)\right\|<r$ and $\left\langle x-S\left(u_{o}\right), T(x)>\geq 0\right.$, for all $x \in K$ satisfying $r \leq\|x\| \leq \max \left(r, r_{o}\right)$ where $r_{o}$ is a number such that $\sup \left\{\|S(u)\| \mid u \in K_{r} \leq\right\} \leq r_{o}$,

$\left.3^{\circ}\right)<S(x), T(x)>\leq\left\langle x, T(x)>; \forall x \in K_{r} \leq\right.$,

then the problem $I C P(T, S, K)$ has a solution $x_{*} \in K_{r} \leq$ such that $\left\|S\left(x_{*}\right)\right\| \leq \max \left(r, r_{o}\right)$. 
PROOF. Since $K$ is locally compact we have that $K_{r} \leq$ is a convex compact set. Applying Theorem 2 with $D=K_{r} \leq$, we obtain an element $x_{*} \in K_{r} \leq$ such that

$$
(3):<x-S\left(x_{*}\right), T\left(x_{*}\right)>\geq 0 ; \forall x \in K_{r} \leq
$$

We have that $S\left(x_{*}\right) \in K$. Two cases are possible:

I) $\left\|S\left(x_{*}\right)\right\|<r$. If $x \in K$ is an arbitrary element then there is a sufficiently small $\left.\lambda \in\right] 0,1[$ such that $w=\lambda x+(1-\lambda) S\left(x_{*}\right) \in K_{r} \leq$. If in (3) we put $x=w$ we have, $\lambda<x-S\left(x_{*}\right), T\left(x_{*}\right)>\geq 0$, that is, $\left\langle x-S\left(x_{*}\right), T\left(x_{*}\right)>\geq 0\right.$ for all $x \in K$ and by Proposition 1 we obtain that $x_{*}$ is a solution of the problem $I C P(T, S<\mathrm{K})$.

II) $\left\|S\left(x_{*}\right)\right\| \geq r$. In this case we have $r \leq\left\|S\left(x_{*}\right)\right\| \leq \max \left(r, r_{0}\right)$ and by assumption $\left.2^{\circ}\right)$ we obtain,

$$
(4):<S\left(x_{*}\right)-S\left(u_{o}\right), T\left(x_{*}\right)>\geq 0,
$$

and since for every $x \in K_{r} \leq$ we have

$$
\text { (5): }\left\langle x-S\left(x_{*}\right), T\left(x_{*}\right)>\geq 0\right.
$$

we deduce (using (4) and (5)), $\left\langle x-S\left(u_{o}\right), \quad T\left(x_{*}\right)\right\rangle=\left\langle x-S\left(x_{*}\right)+S\left(x_{*}\right)-S\left(u_{o}\right), \quad T\left(x_{*}\right)\right\rangle$ $=\left\langle x-S\left(x_{*}\right), T\left(x_{*}\right)\right\rangle+\left\langle S\left(x_{*}\right)-S\left(u_{o}\right), T\left(x_{*}\right)\right\rangle \geq 0$, that is, we have

$$
(6):\left\langle x-S\left(u_{o}\right), T\left(x_{*}\right)\right\rangle \geq 0 ; \forall x \in K_{r} \leq .
$$

If $x \in K$ is an arbitrary element then there is a sufficiently small $\lambda \in] 0,1[$ such that $v=\lambda x+(1-\lambda) S\left(u_{o}\right) \in K_{r} \leq$. Now, if we put $x=v$ in (6) we obtain,

$$
\text { (7): }\left\langle x-S\left(u_{o}\right), T\left(x_{*}\right)>\geq 0 ; \forall x \in K .\right.
$$

Since $\left\|S\left(u_{o}\right)\right\|<r$ we can put $x=S\left(u_{o}\right)$ in (3) and we deduce,

$$
(8):<S\left(u_{o}\right)-S\left(u_{*}\right), T\left(x_{*}\right)>\geq 0 .
$$

From (7) and (8) we obtain

$$
\text { (9): }\left\langle x-S\left(x_{*}\right), T\left(x_{*}\right)>\geq 0 ; \forall x \in K .\right.
$$

Since $S\left(x_{*}\right) \in K$, from (9) and Proposition 1 we obtain that $x_{*}$ is a solution of the problem $\operatorname{ICP}(T, S, \mathrm{~K})$ and the proof is finished.

Theorem 3 can be extended to Galerkin cones. To obtain this extension we need to introduce a new concept.

We say that $S: K \rightarrow E$ is subordinate to the approximation $\left(K_{n}\right)_{n \in N}$ if there exists $n_{o} \in N$ such that for every $n \geq n_{o}$ we have $S\left(K_{n}\right) \subseteq K_{n}$.

In [13] we indicated some examples of mappings with this property.

Independent of us in [16] is defined the concept of $F$-mapping which is similar to our concept.

In [16] we showed that every $D C$-mapping can be approximated by an $F$-mapping while the class of $D C$-mappings is very reach.

We say that $S: K \rightarrow E$ is r-subordinate to the approximation $\left(K_{n}\right)_{n \in N}$ if there exist $r>0$ and $n_{o} \in N$ such that for every $n \geq n_{o}$ we have $S\left(K_{r} \frac{\leq}{n}\right) \subset K_{n}$, where $K_{r} \frac{\leq}{n}=\left\{x \in K_{n} \mid\|x\| \leq r\right\}$.

REMARK. If $S: K \rightarrow E$ is continuous and r-subordinate to the approximation $\left(K_{n}\right)_{n \in N}$ then $S\left(K_{r} \leq\right) \subset K$.

Indeed, if $x \in K_{r} \leq$ then we have two cases: 
a) $\|x\|<r$. Since $K$ is a Galerkin cone there is a sequence $\left\{x_{n}\right\}_{n \in N}$ such that $x=\lim _{n \rightarrow \infty} x_{n}$ and for every $n \in N, x_{n} \in K_{n}$.

There exists $n_{1} \in N$ such that $\left\|x_{n}-x\right\|<r-\|x\|$, for every $n \geq n_{1}$, which implies, $\left\|x_{n}\right\| \leq\left\|x_{n}-x\right\|+\|x\|<r$.

Since, for every $n \geq \max \left(n_{o}, n_{1}\right)$ we have $S\left(x_{n}\right) \in K_{n} \subset K$, we obtain by continuity that $S(x) \in K$. b) $\|x\|=r$. If for every $n \in N, x_{n} \in K_{n} \lim _{n \rightarrow \infty} x_{n}=x$ and $r<\left\|x_{n}\right\|$ then considering the sequence $\underset{\text { and }}{y_{n}=}\left(\frac{r}{\left\|x_{n}\right\|}-\varepsilon_{n}\right) x_{n}$, where $0<\varepsilon_{n}<\frac{r}{\left\|x_{n}\right\|} ; \forall n \in N$ and $\lim _{n \rightarrow \infty} \varepsilon_{n}=0$ we have that $y_{n} \in K_{n},\left\|y_{n}\right\|<r$ $\lim _{n \rightarrow \infty} y_{n}=x$, which imply that $S(x)=\lim _{n \rightarrow \infty} S\left(y_{n}\right) \in K$.

THEOREM 4. Let $(E,\|\|)$ be a reflexive Banach space and $K(K n) n \in N$ a Galerkin cone in $E$. Let $S: K \rightarrow E$ and $T: K \rightarrow E^{*}$ be strongly continuous mappings.

If the following assumptions are satisfied:

$1^{\circ)} \quad S$ is $r$-subordinate to the approximation $\mathrm{K}(\mathrm{K} n) n \in N$,

$\left.2^{\circ}\right)$ there exist $m \in N$ and $u_{o} \in K_{m}$ such that $\left\|S\left(u_{o}\right)\right\|<r, \quad S\left(u_{o}\right) \in K_{m}$ and $<x-S\left(u_{o}\right)$, $T(x)>\geq 0$, for all $x \in K_{n}$ satisfying $r \leq\left\|x_{n}\right\| \leq \max \left(r, r_{n}\right)$, where $r_{n}$ is a number such that $\sup \left\{\|S(u)\| \mid u \in K_{n} \leq\right\} \leq r_{n}$ and for every $n \geq \max \left(n_{o}, m\right)$,

$\left.\left.3^{\circ}\right)<S(x), T(x)\right\rangle \leq\left\langle x, T(x)>; \forall x \in K_{r} \leq\right.$, then the problem $\operatorname{ICP}(T, S, \mathrm{~K})$ has a solution $x_{*}$ such that $\left\|x_{*}\right\| \leq r$.

PROOF. We remark that for every $n \geq \max \left(n_{o}, m\right)$ the all assumptions of Theorem 3 are satisfied for every problem $I C P\left(T, S, \mathrm{~K}_{n}\right)$ and hence we have a solution $x_{n}^{*}$ for each of these problems.

Since for ever $x_{n}^{*}\left(\right.$ with $n \geq \max \left(n_{o}, m\right)$ ) we have $\left\|x_{n}^{*}\right\| \leq r$ we have that $\left\{x_{n}\right\}_{n \in N}$ is a bounded sequence.

Because $E$ is reflexive $\left\{x_{n}^{*}\right\}_{n \in N}$ has a weakly convergent subsequence $\left\{x_{n k}^{*}\right\}_{k \in N}$. We denote again this subsequence by $\left\{x_{n}^{*}\right\}_{n \in N}$ and we put $x_{*}=(w)-\lim _{n \rightarrow \infty} x_{n}^{*}$. We have that $x_{*} \in K$ and $\left\|x_{*}\right\| \leq r$, since $K_{r} \leq$ is closed and convex. Hence $S\left(x_{*}\right) \in K$.

Let $x \in K$ be an arbitrary element. For every $n \geq \max \left(n_{o}, m\right)$ we have,

$$
(10):\left\langle P_{n}(x)-S\left(x_{n}^{*}\right), T\left(x_{n}^{*}\right)>\geq 0,\right.
$$

where $\left\{P_{n}\right\}_{n \in N}$ is a sequence of projections. Since $S$ and $T$ are strongly continuous, computing the limit in (10) we obtain,

$$
(11):<x-S\left(x_{*}\right), T\left(x_{*}\right)>\geq 0 \text {; for all } x \in K .
$$

The proof is finished since from (11) by Proposition 1 we have that $x_{*}$ is a solution of the problem $I C P(T, S, \mathrm{~K})$.

We consider now the case when $S(K) \subseteq K$.

THEOREM 5. Let $(E,\|\|)$ be a Banach space, $K \subset E$ a pointed locally compact convex cone and $S: K \rightarrow K, T: K \rightarrow E^{*}$ continuous mappings.

If the following assumptions are satisfied:

$\left.\left.1^{\circ}\right)<S(x), T(x)\right\rangle \leq\langle x, T(x)>; \forall x \in K$,

$\left.2^{\circ}\right)$ there is $r>0$ such that for every $x \in K$ with $r \leq\|x\|$ there is an element $v_{x} \in K$ such that $\left\|v_{x}\right\|<r$ and $\left\langle S(x)-v_{x}, T(x)>>0\right.$, then the problem $\operatorname{ICP}(T, S, K)$ has a solution $x_{*}$ such that $\left\|x_{*}\right\|<r$.

PROOF. We denote $D_{n}=\{x \in K \mid\|x\| \leq n\}$. Since $K$ is locally compact we have that for every $n \in N, D_{n}$ is a convex compact set.

We apply Theorem 2 with $D=D_{n}$ and we obtain a solution $x_{n}^{*}$ for the problem $\operatorname{SVI}\left(T, S, D_{n}\right)$ 
So we have:

$$
\text { (12): }\left\{\begin{array}{l}
\text { for every } n \in N \text { there is } x_{n}^{*} \in D \text { such that } \\
\left\langle S\left(x_{n}^{*}\right)-v, T\left(x_{n}^{*}\right)>\leq 0 ; \forall v \in D_{n}\right.
\end{array}\right.
$$

The sequence $\left\{x_{n}^{*}\right\}_{n \in N}$ is bounded. Indeed, supposing the contrary we have $(\forall k>0)(\exists n \in N)\left(\left\|x_{n}^{*}\right\| \geq k\right)$.

If $k \geq r$ then there is a natural number $n$ such that, $n \geq\left\|x_{n}^{*}\right\| \geq k \geq r$. For this $x_{n}^{*}$, by assumption $2^{\circ}$ ) there is an element $v_{x^{*}{ }_{n}} \in K$ such that $\left\|v_{x^{*}}{ }_{n}\right\|<r$ and,

$$
\text { (13): }<S\left(x_{n}^{*}\right)-v_{x_{n}^{*}}, T\left(x_{n}^{*}\right)>>0 .
$$

But, since $\left\|v_{x^{*}}{ }_{n}\right\|<r<\left\|x_{n}^{*}\right\| \leq n$, from (12) we have $<S\left(x_{n}^{*}\right)-v_{x^{*}}{ }_{n}, T\left(x_{n}^{*}\right)>\leq 0$, which is a contradiction of (13).

Hence, $\left\{x_{n}^{*}\right\}_{n \in N}$ is bounded and because $K$ is locally compact the sequence $\left\{x_{n}^{*}\right\}_{n \in N}$ has a norm convergent subsequence $\left\{x_{n_{k}}^{*}\right\}_{k \in N}$.

Let $x_{*}=\lim _{k \rightarrow \infty} x_{n_{k}}^{*}$. We show now that $x_{*}$ is a solution of the problem $\operatorname{ICP}(T, S, K)$.

Indeed, if $v \in K$ is an arbitrary element, then there is $m \in N$ such that for every $n \geq m$ we have, $v \in D_{n}$ and for every $n_{k} \geq m, v \in D_{n_{k}}$ and $\left\langle S\left(x_{n_{k}}^{*}\right)-v, T\left(x_{n_{k}}^{*}\right)\right\rangle \leq 0$.

Using the continuity of $S$ and $T$ we obtain, $\left\langle S\left(x_{*}\right)-v, T\left(x_{*}\right)>\leq 0, \forall v \in \mathrm{K}\right.$, that is $x_{*}$ is a solution of the problem $S V I(T, S, \mathrm{~K})$ which, by Proposition 1 is equivalent to the problem $\operatorname{ICP}(T, S, K)$. Obviously, by assumption $\left.2^{\circ}\right)$ we must have $\left\|x_{*}\right\|<r$.

From Theorem 5 we deduce two important corollaries.

COROLLARY 1. Let $K \subset E$ be a pointed locally compact cone and $S: K \rightarrow K, T: K \rightarrow E^{*}$ continuous mappings. If the following assumptions are satisfied:

$\left.\left.1^{\circ}\right)<S(x), T(x)\right\rangle \geq\langle x, T(x)\rangle ; \forall x \in K$,

$2^{\circ}$ ) there is a number $r>0$ such that for every $x \in K$ with $r \leq\|x\|$ we have $<S(x), T(x)>>0$,

then the problem $\operatorname{ICP}(T, S, \mathrm{~K})$ has a solution $x_{*}$ such that $\left\|x_{*}\right\| \leq r$.

PROOF. We apply Theorem 5 with $v_{*}=0$ for every $x \in K$ satisfying $\|x\| \geq r$.

COROLLARY 2. Let $K \subset E$ be a pointed locally compact cone and $S: K \rightarrow K, T: K \rightarrow E^{*}$ continuous mappings. If the following assumptions are satisfied:

$1^{\circ)}\langle S(x), T(x)\rangle \leq\langle x, T(x)>; \forall x \in K$,

$\left.2^{\circ}\right)$ there is a number $r_{o} \geq 0$ and $u_{o} \in K$ such that for every $x \in K$ with $r_{o} \leq\|x\|$ we have $<S(x)-u_{o}, T(x)>>0$.

then the problem $I C P(T, S, K)$ has a solution $x_{*}$ such that $\left\|x_{*}\right\|<1+\max \left(r_{o},\left\|u_{o}\right\|\right)$.

PROOF. If we denote, $r=\max \left(r_{o},\left\|u_{o}\right\|\right)+1$, we have $r>r_{o}$ and $r>\left\|u_{o}\right\|$.

Now, we can apply Theorem 5 since assumption $2^{\circ}$ ) of this theorem is satisfied with $v_{x}=u_{o}$, for every $x \in K$ with $\|x\| \geq r$.

REMARK. Condition $2^{\circ}$ ) of Corollary 2 is satisfied if $T$ is semicoercive with respect to $S$ in the following sense:

$$
\left(\exists u_{o} \in K\right)\left(\lim _{\|x\| \rightarrow \infty} \frac{<S(x)-u_{o}, T(x)>}{\|x\|}=+\infty\right)
$$


If $S(x)=x$, for every $x \in K$, this notion is similar to the semicoercivity used by Stampacchia and Lions [22], [23].

Obviously, condition $2^{\circ}$ ) is satisfied if there is a number $\alpha>0$ such that $<S(x)$, $T(x)>\geq \alpha\|x\|^{2}$, for every $x \in K$.

Finally, we give an extension of Theorem 5 to Galerkin cone.

THEOREM 6. Let $(E,\|\|)$ be a reflexive Banach space and $K\left(K_{n}\right)_{n \in N}$ a Galerkin cone in $E$. Let $S: K \rightarrow K$ and $T: K \rightarrow E^{*}$ be strongly continuous mappings.

If the following assumptions are satisfied:

$1^{\circ)} S$ is subordinate to the approximation $(K)_{n} \in N$,

$\left.2^{\circ}\right)<S(x), T(x)>\leq<x, T(x)>; \forall x \in K$,

$\left.3^{\circ}\right)$ there is a number $r>0$ such that for every $n \geq n_{o}$ and every $x \in K_{n}$ with $r \leq\|x\|$ there is an element $v_{x} \in K_{n}$ such that $\left\|v_{x}\right\|<r$ and $\left\langle S(x)-v_{x}, T(x)>>0\right.$, then the problem $\operatorname{ICP}(T, S, \mathrm{~K})$ has a solution $x_{*}$ such that $\left\|x_{*}\right\| \leq r$.

PROOF. Since, for every $n \geq n_{o}$ we have $S\left(K_{n}\right) \subseteq K_{n}$ and the all assumptions of Theorem 5 are satisfied, we have that for every $n \geq n_{o}$ the problem $\operatorname{ICP}\left(T, S, \mathrm{~K}_{n}\right)$ has a solution $x_{n}^{*}$.

Because for every $n \geq n_{o}$ we have $\left\|x_{n}^{*}\right\|<r$ and $E$ is reflexive the sequence $\left\{x_{n}^{*}\right\}_{n \in N}$ has a weakly convergent subsequence denoted again by $\left\{x_{n}^{*}\right\}_{n \in N}$. We put $x_{*}=(w)-\lim _{n} x_{\infty} x_{n}^{*}$.

Now, as in the proof of Theorem 4 we conclude that $x_{*}$ is a solution of the problem $I C P(T, S, \mathrm{~K})$. Obviously, $\left\|x_{*}\right\| \leq r$.

ACKNOWLEDGEMENT. The authors thank Professor V.H. Nguyen for several helpful comments on the first version of the paper.

\section{REFERENCES}

1. BERSHCHANSKII, Y.M. \& MEEROV, M.T., The complementarity problem: Theory and methods of solution, Automat. Remote Control 44 Nr. 6 (1983), 687-710.

2. BENSOUSSAN, A., ET LIONS, J.L., Nouvelle formulation de problémes de contrôle impulsionnel et applications, C.R. Acad. Sci. Paris Sér. A-B 276 (1973), 1189-1192.

3. BENSOUSSAN, A., ET LIONS, J.L., Nouvelle formulation de problémes de contrôle impulsionnel, Applied Mathematics and Opt. 1 (1974), 289-312.

4. BENSOUSSAN, A., GOURSET, M. ET LIONS, J.L., Contrôle impulsionnel et inéquations quasi-variationnelle stationnaries, C.R. Acad. Sci. Paris Sér A-B 276 (1973), 1279-1284.

5. COTTLE, R.W., Complementary and variational problems, Symposia Mathematicae 19 (1976), 177-208.

6. DOLCETTA, I.C., LORENZANI, M. \& SPIZZICHINO, F., A degenerate complementarity system and applications to the optimal stopping of Markov chains, Boll. U.I. (5) 17-B (1980), 692-703

7. DOLCETTA, I.C., \& MOSCO, U., Implicit complementarity problems and quasi-variational inequalities, Variational Inequalities and Complementarity Problems Theory and Applications (Eds. R.W. Cottle, F. Giannessi and J.L. Lions) John Wiley \& Sons, New York, London (1980), 271-283.

8. ISAC, G., Problémes de complémentarité (En dimension infinie) (Minicours), Publ. Dépt. Math. Univ. Limoges, France (1985).

9. ISAC, G., Complementarity problem and coincidence equations on convex cones, Bull. Un. Mat. Ital. B(6)5 (1986), 925-943.

10. ISAC, G., Nonlinear complementarity problem and Galerkin method, J. Math. Anal. Appl. 108 (1985), 563-574.

11. ISAC, G., Fixed point theory and complementarity problems in Hilbert space, Bull. Austr. Math. Soc. 36 Nr. 2 (1987), 295-310. 
12. ISAC, G., Fixed point theory, coincidence equations on convex cones and complementarity problem, Contemporary Math. 72 (1988), 139-155.

13. ISAC, G., A special variational inequality and the implicit complementarity problem, J. Fac. Science Univ. Tokyo Sec. 1A 37 Nr. 1 (1990), 109-127.

14. ISAC, G., \& KOSTREVA, M., The generalized order complementarity problem, J. Opt. Theory Appl. 17 No. 3 (1991), 517-534.

15. KARAMARDIAN, S., Generalized complementarity problem, J. Optim. Theory Appl. 8 Nr. 3 (1971), 161-168.

16. KRYSZEWSKI, W. \& PRZERADZKI, B., The topological degree and fixed point of DCmappings, Fundamenta Mathematicae 126 (1985), 15-26.

17. LIPKIN, L.J., Weak continuity and compactness of nonlinear mappings, Nonlinear Anal. Theory, Method, and Appl. 5 Nr. 11 (1981), 1257-1263.

18. MOSCO, U., Implicit variational problems and quasi-variational inequalities, Lecture Notes in Math. Vol. 543, Springer-Verlag, Berlin (1976).

19. MOSCO, U., On some nonlinear quasi-variational inequalities and implicit complementarity problems in stochastic control theory, Variational Inequalities and Complementarity Problems. Theory and Applications, (Eds, R.W. Cottle, F. Giannessi and J.L. Lions), John Wiley \& Sons Chichester, (1980), 75-87.

20. PANG, J.S., The implicit complementarity problem, Nonlinear Programming IV (Eds, O.L. Mangasarian, R.R. Meyer and S.M. Robinson) Academic Press, New York, London. (1981), 487-518.

21. PANG, J.S., On the convergence of a basic iterative method for the implicit complementarity problem, J. Optim. Theory Appl. 37 (1982), 149-162.

22. STAMPACCHIA, G., Variational inequalities, Theory and Applications of Monotone Operators (Ed. A. Ghizetti), Edizione Odersi, Gubio (1969), 101-192.

23. STAMPACCHIA, G., \& LIONS, J.L., Variational inequalities, Comm. Pure Appl. Math. 20 (1967), 493-519.

24. VAINBERG, M.M., Variational methods for the study of nonlinear operators, Holden-Day Inc., San Francisco, London, Amsterdam (1964).

25. ZEIDLER E., Nonlinear functional analysis and applications, Tom 1 Fixed Point Theory, Springer-Verlag (1985). 


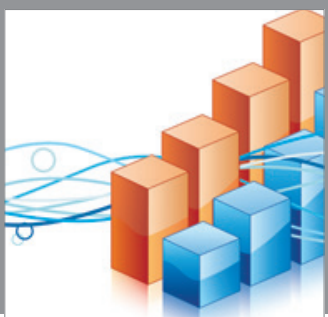

Advances in

Operations Research

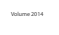

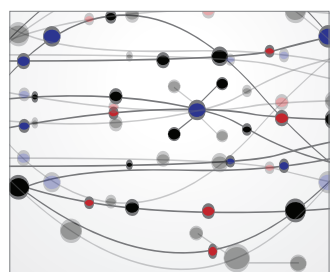

\section{The Scientific} World Journal
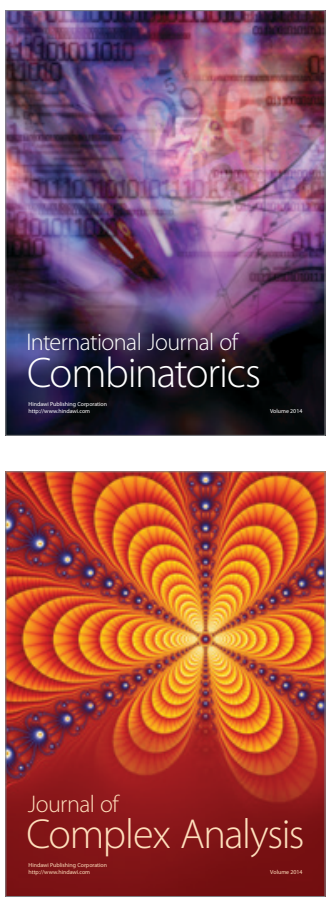

International Journal of

Mathematics and

Mathematical

Sciences
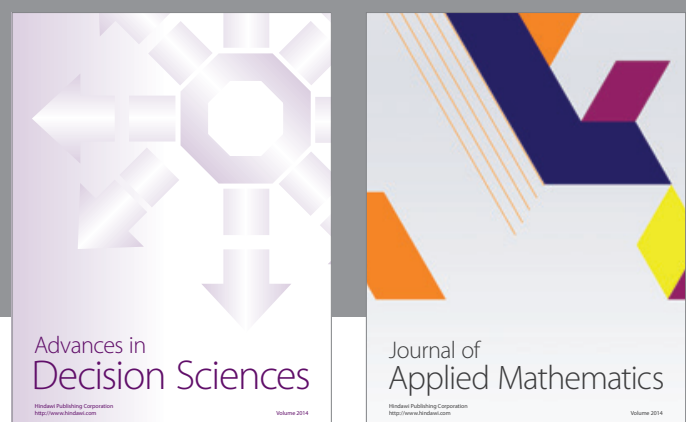

Journal of

Applied Mathematics
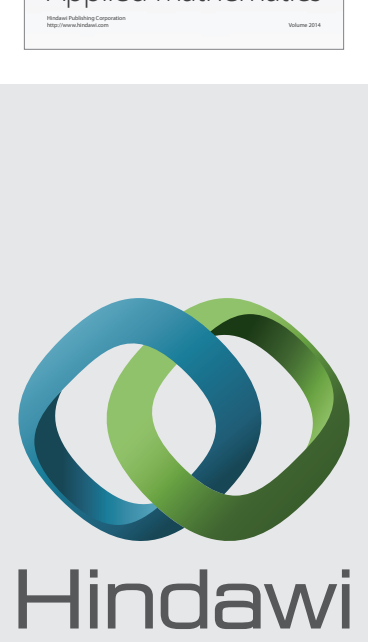

Submit your manuscripts at http://www.hindawi.com
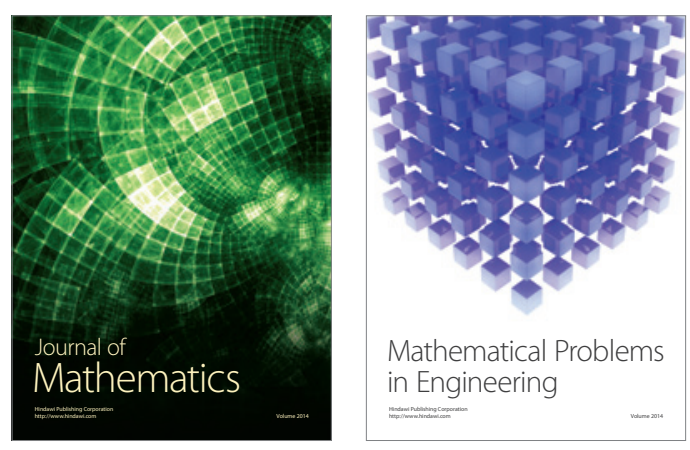

Mathematical Problems in Engineering
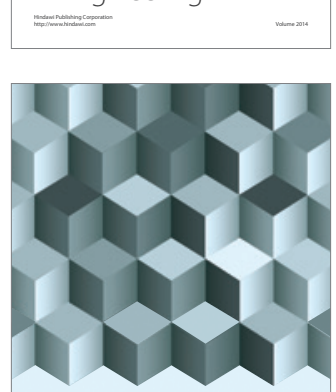

Journal of

Function Spaces
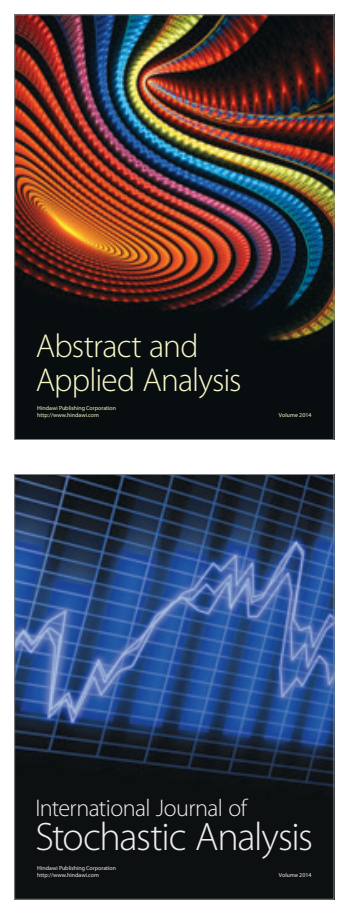

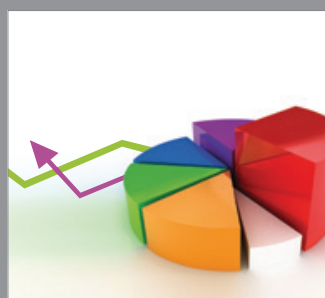

ournal of

Probability and Statistics

Promensencen
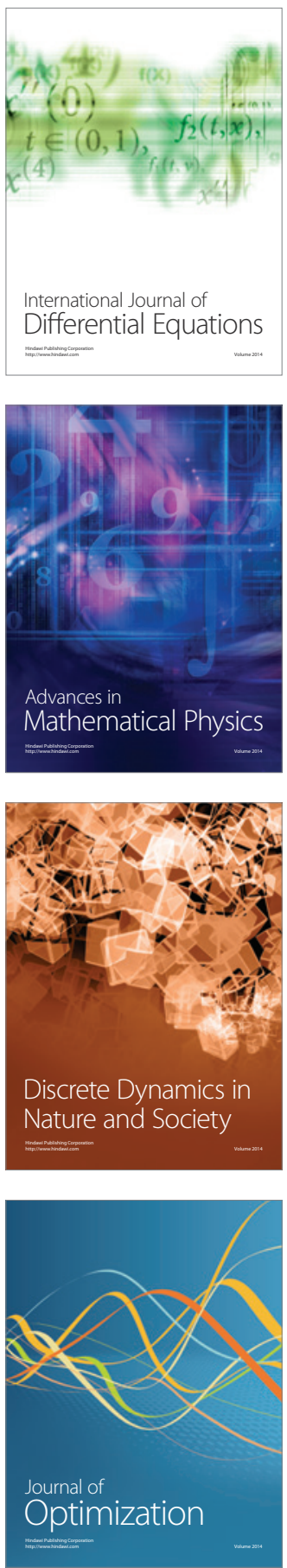